\title{
Surfactant-Free One-Step Synthesis of Dual-Functional Polyurea Microcapsules: Contact Infection Control and Drug Delivery
}

\author{
Wei He, Xiaochen Gu, and Song Liu*
}

Surface functionalized polyurea microcapsules (MCQ) are synthesized in one step. Dimethyl-dodecyl-(5-hydroxy-pentyl)-ammonium bromide (DAB), a hydroxyl-end-capped quaternary ammonium salt, is synthesized and adopted as a new surfmer for the synthesis of MCQ. It is confirmed by fluorescein adsorption that DAB is covalently bonded to MCQ. The so-formed MCQ possess dual-functionality: contact infection control and sustained drug delivery. Agar diffusion antimicrobial tests confirm successful inhibition of multidrug-resistant $E$. coli by MCQ alone instead of by leaching of free quaternary ammonium salts. Furthermore, few E. coli colonies survive on an agar plate coated with 3-4 layers of MCQ. Dissolution tests show a typical first-order release profile of courmarin-1, a model dye, from MCQ.

\section{Introduction}

Antimicrobial microcapsules are generally based on the release of encapsulated cytotoxic agents. ${ }^{[1-3]}$ Cytotoxic agents have been encapsulated for various reasons. For the purpose of maintaining an effective therapeutic concentration, the antibacterial drug succinylsulphathiazole was trapped in biodegradable polyphosphazene microcapsules to achieve its controlled release during the healing period of periodontal defects (around one month). ${ }^{[1]}$ A water-insoluble unstable natural preservative, curcumin, was encapsulated to ensure long-term and effective antimicrobial activity in the food processing industry. ${ }^{[2]}$ Carvacrol, an antimicrobial essential oil, was enveloped in Ca-alginate to avoid its early absorption in the upper gastrointestinal tract during delivery to the small intestine. ${ }^{[3]}$

W. He, Dr. S. Liu

Department of Textile Sciences

Faculty of Human Ecology

University of Manitoba

Winnipeg, Manitoba R3T 2N2, Canada

E-mail: lius0@cc.umanitoba.ca

Dr. X. Gu

Faculty of Pharmacy

University of Manitoba

Winnipeg, Manitoba R3E 0T5, Canada

Dr. S. Liu

Department of Chemistry

Faculty of Science

University of Manitoba

Winnipeg, Manitoba R3T 2N2, Canada
Sustained release of antibiotics suffers from the major disadvantage of possible occurrence of antibiotic resistance. Even for silver based biocidal agents whose antimicrobial mechanism is believed to arise from chemical interactions with sulfydryl $(-\mathrm{SH})$ groups of proteins, ${ }^{[4,5]}$ there have been several reports of induced bacterial resistance. ${ }^{[6-8]}$ Recently, non-leaching on-contact biocidal microcapsules have attracted interest from investigators. Cui et al. ${ }^{[9]}$ fabricated microcapsules with antimicrobial chitosan and hyaluronic acid via the polyelectrolyte multilayer (PEM) deposition, and endowed the drug carrier with an inherently antimicrobial shell. Contact killing offers benefits such as long lasting biocidal effect and no induced biocide resistance. With the contact killing shell in place, the interior of the microcapsules can be used to envelop other bioactive reagents, yielding multifunctional microcapsules that are highly useful in medical applications. For example, in addition to an antibacterial function, an anti-inflammatory function should also be delivered in the treatment of periodontal diseases since surgical treatment can cause painful inflammation. If an anti-inflammatory drug were encapsulated in the inherently antibacterial microcapsules, the combination of two functions could be achieved. In spite of the trend of imparting microcapsules with on-contact biocidal functions, the potential dual functions of drug release and antibacterial activity has never been explored.

Interfacial polymerization is a facile, convenient and fast way of synthesizing microcapsules. Polyurea microcapsules (PUMCs) can be formed by the interfacial polymerization of isocyanates and amines. We chose to work with PUMC because its formation is fast and scalable; its monomers are flexible enough to allow for the introduction of surface functions, and the synthesis is compatiable with any type of emulsion: water-inoil, ${ }^{[10]}$ oil-in-water, ${ }^{[11]}$ and the relatively new oil-in-oil. ${ }^{[12]}$ Current strategies for surface modification of PUMCs are categorized into post-modification and in-situ modification. By making use of the positive charges $\left(-\mathrm{NH}_{3}{ }^{+}\right)$on the external surfaces of PUMCs synthesized in an oil-in-water system, Stover and coworkers ${ }^{[13]}$ post-modified the PUMCs with an ionic pair, anionic delaminated montmorillonite clay and a polycation poly $(N$ [3-aminopropyl]-methacrylamide hydrochloride), to control the release of the encapsulated volatile model compound xylene. To overcome the inherent instability of the polyelectrolyte coating,

DOI: $10.1002 / \mathrm{adfm} .201200656$ 


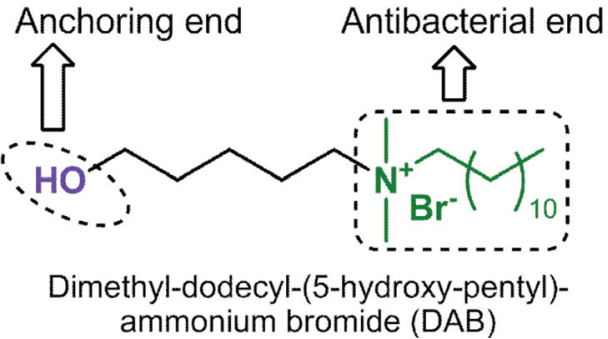

Scheme 1. Structures of dimethyl-dodecyl-(5-hydroxy-pentyl)-ammonium bromide (DAB, an antibacterial cationic surfactant ended with a hydroxyl group) and 2-azidoethylamine (AEA, a labelling agent for active isocyanate).

a polyanion containing amine reactive acetoacetyl groups was covalently anchored onto PUMCs. ${ }^{[14]}$ Even though postmodification methods possess the advantage of minimal interference with the shell formation of PUMCs, the long preparation route may impede their large scale applications. For in-situ modification, functional monomers were incorporated into the shell during interfacial polyaddition. ${ }^{[10,11]} 4,4^{\prime}$-diaminostibene2,2'-disulfonic acid (DASSA) was used as a comonomer during the synthesis of PUMCs in a water-in-oil system to improve their oxygen affinity as potential red blood cell substitues. ${ }^{[10]}$ To improve the water dispersibility of PUMCs, lysine was incorporated into the PUMC shells during the synthesis in an oil-inwater system. Both reports adopted surfactants to get PUMCs in the size range of 8-70 $\mu \mathrm{m}$. Some drugs are soluble neither in water nor in non-polar solvents and thus cannot be efficiently enveloped by PUMC using the conventional oil-in-water or water-in-oil systems. The recently reported oil-in-oil interfacial synthesis of PUMC ${ }^{[12]}$ has provided a useful tool to encapsulate these drugs. Nonetheless, PUMC synthesized in a surfactantfree oil-in-oil system with a functional surface has not been reported.

In this study, we proposed a surfactant-free in-situ modification to impart PUMC with on-contact antibacterial function. It was reported that during the synthesis of PUMC, residue isocyanate groups on the shell had to be quenched in an extra step. ${ }^{[15]}$ Isocyanate is highly reactive with nucliphiles such as hydroxyl and amino groups. It was hypothesized that new functions such as a nonleaching antimicrobial function could be quickly and conveniently introduced by making use of the residue reactive isocyanate group on the shell of PUMC synthesized using an oil-in-oil system. A new quaternary ammonium salt with hydroxyl group, (dimethyl-dodecyl-(5-hydroxy-pentyl)ammonium bromide, DAB, Scheme 1), was synthesized for the functional modification of PUMC. We first attemped a post-modification method. The pre-formed $2 \mu \mathrm{m}$ narrow sized PUMC, however, had poor dispersibility in water making the susequent modification troublesome. The surfactant (sorbitane trioleate, Span 85) used in the synthesis was believed to be actually grafted onto PUMC shells, leading to a hydrophobic PUMC shell having poor water dispersibility. This finding inspired us to adopt amphiphilic DAB as a surfmer in the interface of immiscible oils (such as cyclohexane and dimethylformamide (DMF)) for one-step synthesis of an inherently antibacterial PUMC. The antibacterial and drug release properties of the surface modified PUMC were evaluated. with free PEI inside the microcapsule. As a result, the PUMC shell grew inward instead of outward since PEI is insoluble in the continuous phase cyclohexane. Given that excess amount of TDI (at least 9 times in excess compared with available amine groups) was used in the experiment, a considerable amount of free isocyanate group from mono-reacted TDI was expected to become available on the outer surface of the primary membrane (Scheme 2). We hypothesized that surface isocyanate groups on the shell could be used for functionalization of PUMC by anchoring hydroxyl or amine-ended functional molecules. To test this hypothesis, we first experimented with a model system (polyurea macrocapsules) without the emulsification process as a proof of concept. Subsequently, emulsification was conducted to synthesize inherently antibacterial polyurea microcapsules. Finally, antimicrobial and drug delivery properties of the antibacterial polyurea microcapsules were assessed.

\subsection{Synthesis and Characterization of model Polyurea Macrocapsules (PUCs)}

PUC was synthesized without surfactant or thickener to eliminate the washing process and to avoid possible loss of active isocyanate groups. 2-azidoethylamine (AEA, Scheme 1) was synthesized to serve as a labelling reagent with an anchoring end (amine group that can react with residue isocyanate groups) and a reporting end (azide group possessing a high infrared

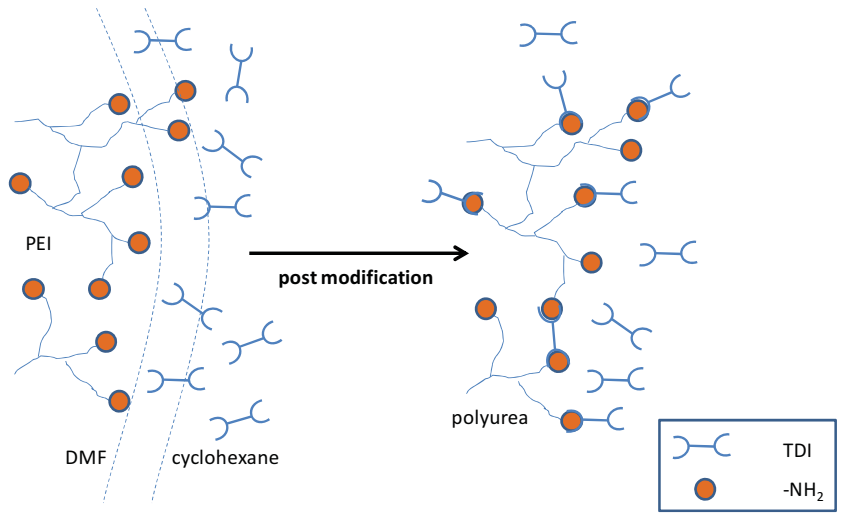

Scheme 2. Free isocyanate group on the surface of polyurea microcapsules available for further modifications. 


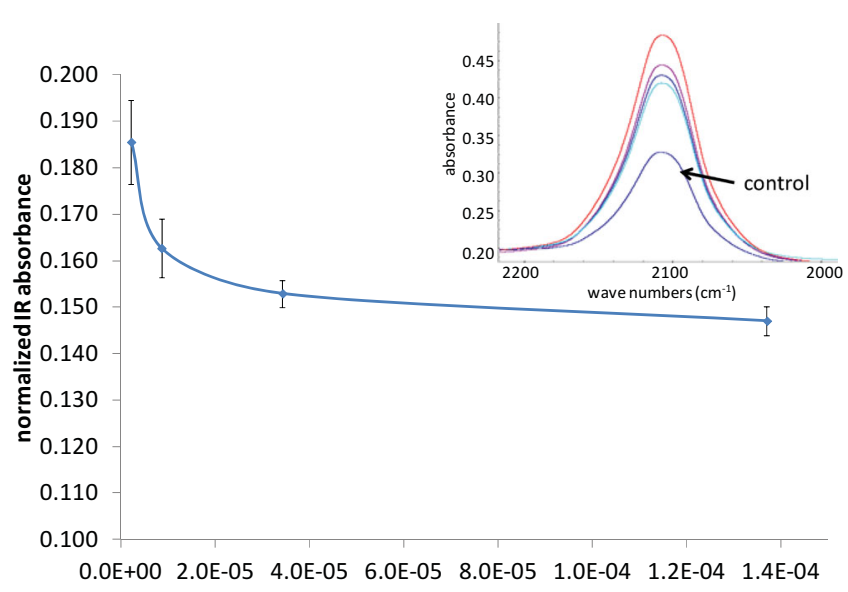

concentration of ethanol $\left(\mathrm{mol} \mathrm{L}^{-1}\right)$

Figure 1. IR absorbance at $2108 \mathrm{~cm}^{-1}$ (peak heights) of AEA (2-azidoethylamine) labelled model macrocapsules (PUCs) as a function of the concentration of hexane solution of ethanol used in the step of quenching. The original spectra are shown in the inset.

molar extinction coefficient). Two strategies could be utilized to monitor the relative amount of residue isocyanate: 1) change the dosage of TDI in PUC synthesis and label the residue isocyanate using AEA afterwards and 2) to use varying amounts of ethanol to partially quench residue isocyanate on the shell of freshly prepared PUC and then label the unquenched isocyanate with AEA. The first approach might experience difficulty in managing AEA rinsing cycles; therefore, the second strategy was adopted in this study. Freshly prepared PUC was partially quenched with ethanol and then labelled with AEA. Afterwards, AEA labelled PUCs were subjected to IR examination. The IR absorbance at $2108 \mathrm{~cm}^{-1}$ (azide absorption) was normalized with amide I band which is predominant in PUC.

Figure 1 and Table 1 show the original spectra and processed data of AEA labelled PUC, respectively. Normalized IR absorbance represented the active isocyanate group in the shell after partial quenching. A fast initial drop (from A to C) followed by a plateau (from $\mathrm{C}$ to $\mathrm{D}$ ) of surface isocyanate groups was observed upon the introduction of a small amount of ethanol $\left(<3.4 \times 10^{-5} \mathrm{~mol} \mathrm{~L}^{-1}\right)$, which suggested that the surface isocyanate groups were highly sensitive to nucleophiles such as

Table 1. Semi-quantification of residue isocyanate groups on the shell of PUC.

\begin{tabular}{lccc}
\hline Sample & $\begin{array}{c}\text { Conc. of ethanol } \\
{[\mathrm{M}]}\end{array}$ & $\begin{array}{c}\text { Molar ratio of } \\
-\mathrm{OH} / \mathrm{NCO}\end{array}$ & $\begin{array}{c}\text { Normalized IR absorbance } \\
\text { (accessible free-NCO) }\end{array}$ \\
\hline $\mathrm{A}$ & $2.1 \times 10^{-6}$ & 0.02 & $0.186 \pm 0.009(100 \%)$ \\
$\mathrm{B}$ & $8.6 \times 10^{-6}$ & 0.07 & $0.163 \pm 0.006(73 \%)$ \\
$\mathrm{C}$ & $3.4 \times 10^{-5}$ & 0.29 & $0.153 \pm 0.003(62 \%)$ \\
D & $1.4 \times 10^{-4}$ & 1.18 & $0.147 \pm 0.003(55 \%)$ \\
Control & $1.4 \times 10^{-3}$ & 11.8 & $0.100 \pm 0.009(0 \%)$ \\
\hline
\end{tabular}

a) Normalized IR absorbance is presented by the average of 3 samples \pm standard deviation. An estimation of accessible free isocyanate groups in the shell is presented in brackets. hydroxyl groups, and surface modification of PUC was possible by taking advantage of isocyanate residue on the shell. For the control sample, in which all free isocyanate groups were supposed to be quenched with ethanol, the signal was attributed to the physically adsorbed AEA that was unable to be removed completely by repeated extraction. We can assume all free isocyanate groups were quenched in the control sample and all free isocyanate groups were labelled by AEA in sample A (Table 1) since only negligible isocyanate groups were quenched considering low molar ratio of ethanol $-\mathrm{OH}$ to theoretical residue isocyanate groups (0.02). Based on this assumption, accessible isocyanate groups on the PUC shell for all the samples (A-D) could be calculated as percentages listed in Table 1 (in the brackets under the fourth column). A 55\% difference between D and the control sample could be attributed to the active isocyanate group beneath the superficial surface of PUC shell. Higher ethanol concentration $\left(1.4 \times 10^{-4} \mathrm{~mol} \mathrm{~L}^{-1}\right)$ and longer quenching duration (20 min vs. $1 \mathrm{~min}$ ) was adopted in making the control sample to enable ethanol to reach deeper into the shell while in sample A-D, only isocyanate groups on the superfacial surface were quenched. Thus by comparing B to A, we concluded that at least $28 \%$ of free isocyanate groups in the shell of PUC were on the most exterior superficial surface and were suitable for anchoring amine capped molecules.

To test the hypothesis that residue superfacial isocyanate groups on PUC can also anchor a hydroxyl group ended molecule, we grafted the freshly prepared PUC with DAB, a quaternary ammonium salt ended with a hydroxyl group (Scheme 1). The resultant post-modified PUC was referred to as "PUC+Q", where $Q$ stands for the quaternary ammonium salt DAB. DAB on PUC was titrated with fluorescein (as shown in Figure 2). The adsorbed fluorescein on PUC+Q $\left(4.99 \times 10^{-7} \mathrm{~mol} \mathrm{~cm}^{-2}\right)$ was nearly one order of magnitude higher than that of the control sample (PUC, $7.00 \times 10^{-8} \mathrm{~mol} \mathrm{~cm}^{-2}$ ). The quantitative result also corresponded to the staining result: $\mathrm{PUC}+\mathrm{Q}$ demonstrated a deeper color than that of PUC before the extraction

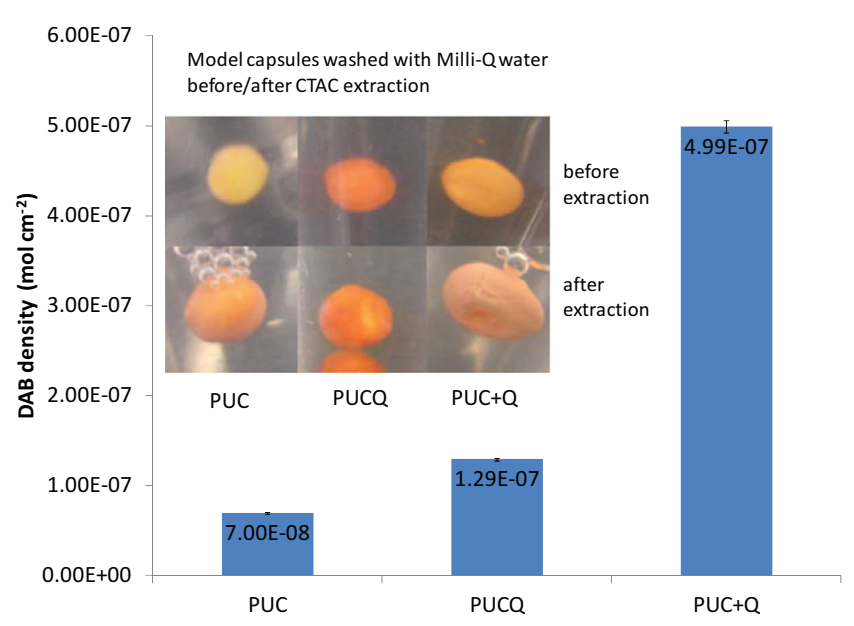

Figure 2. Quantification of DAB density $\left(\mathrm{mol} \mathrm{cm}^{-2}\right)$ on model macrocapsules. Error bar was generated from three batch-to-batch measurements. PUC: control model macrocapsules; PUCQ: in situ modified model macrocapsules; and PUC+Q: post-modified model macrocapsules. 
with a cationic stripping agent, cetyltrimethyl ammoniumchloride (CTAC); the color difference disappeared after extraction (Figure 2 inset). It was hence confirmed that isocyanate residues on the surface of PUC could be preserved and utilized for post-modification in a non-aqueous system at room temperature. We then proceeded to apply the post-modification method to modify PUMC.

\subsection{Synthesis and Characterization of PUMC}

PUMC to be modified with DAB was synthesized according to a protocol adapted from the one previously described. ${ }^{[12]}$ Emulsion stabilized by Span 85 was prepared through magnetic agitation. TDI was introduced immediately after the emulsification process. In order to form a shell that was strong enough for further testings and applications, DMF and methanol were compared as the diluents of the disperse phase.

Methanol was found unsuitable for the preparation. Methanol not only quenched isocyanate groups and caused the formation of by-products (data not shown), but also impeded PEI conversion, especially after the formation of the primary membrane when the shell growth rate was limited by the diffusion rate of TDI rather than the reaction rate. ${ }^{[16]}$ The resultant microcapsules tended to rupture when immersed in water, mainly due to osmotic pressure produced by unreated PEI within the microcapsules. In comparison, DMF was a better candidate as the disperse phase solvent. Nevertheless, microcapsules synthesis from DMF could also suffer from morphological problems. Coexistence of nanocapsules caused severe aggregation of microcapsules (Figure 3a), which eventually led to poor redispersability and low recovery ratio. Formation of nanocapsules was reduced by reducing the concentration of Span 85 (i.e., by reducing the number of formed micelles, Figure $3 \mathrm{~b}$ ). Better results were acheived via encapsulating cortisone, a model hydrophobic drug, to control the coalescence of droplets with $20 \mathrm{~min}$ emulsification interval (drug load = $18 \mathrm{wt} \%$, efficiency $=45 \%$, without optimization, Figure 3c). Cortisone reduces micelle formation by the "salt out" effect. ${ }^{[17,18]}$ Cortisone, in the vicinity of the interface, repels DMF from the interface and reduces the effective hydrophilic head volume of the surfactant, subsequently reducing the interface membrane stability and causing coalescence of nano-droplets. The synthesized PUMC possessed a small mean diameter (volume weighted mean diameter $\left.\left(\mu_{\mathrm{g}}\right)=6.1 \mu \mathrm{m}\right)$ and a narrow size distribution (span value $(\mathrm{S})=0.41$, geometric standard deviation $\left(\sigma_{\mathrm{g}}\right)=1.16$; Figure $\left.3 \mathrm{~d}\right)$.

Even though the morphology problem could be solved, the resultant microcapsules were not very well dispersed in water, and tended to flocculate and float on the surface, indicating that the shell of the synthesized microcapsules was hydrophobic. This hydrophobic nature may be attributed to the grafting of
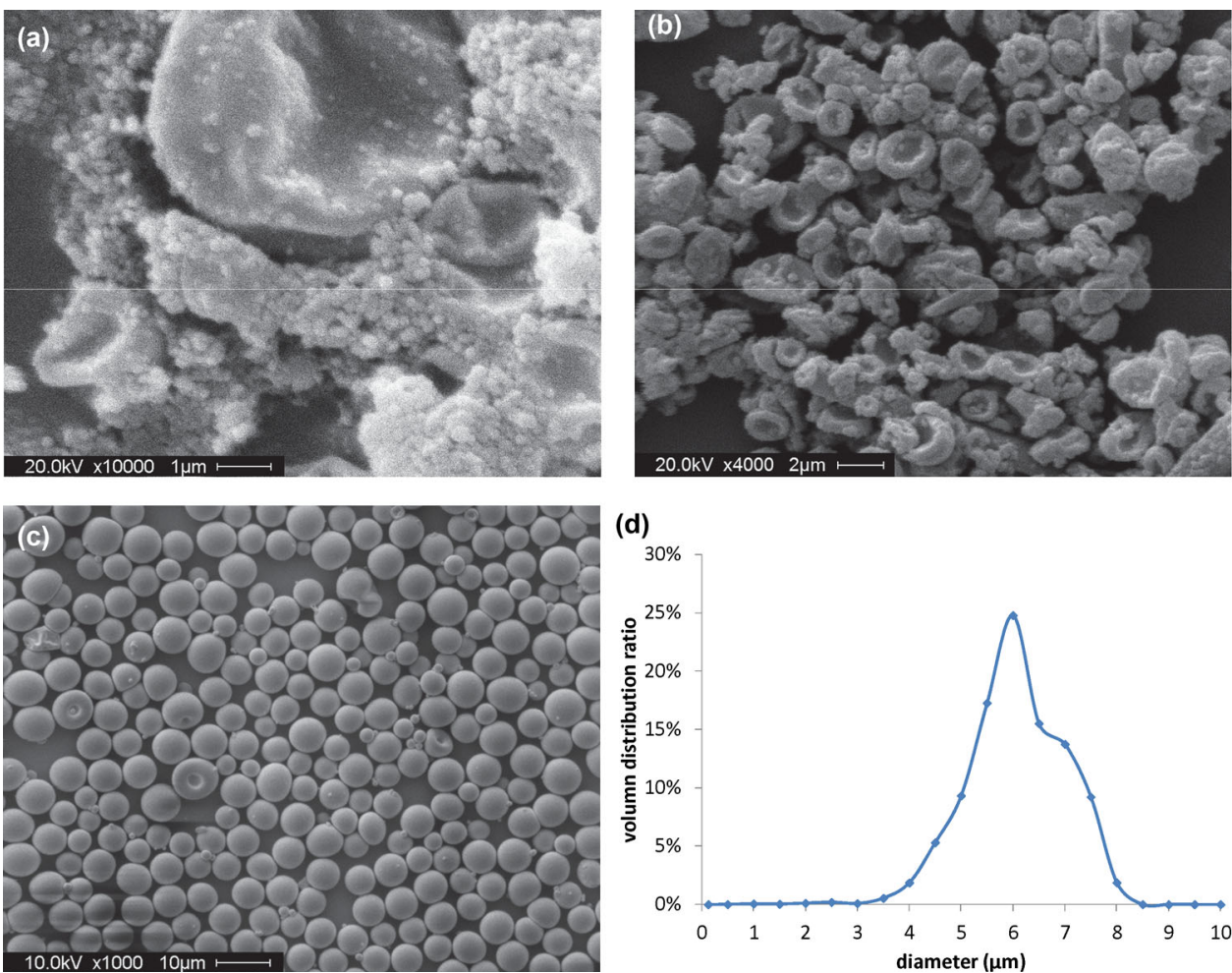

(d)

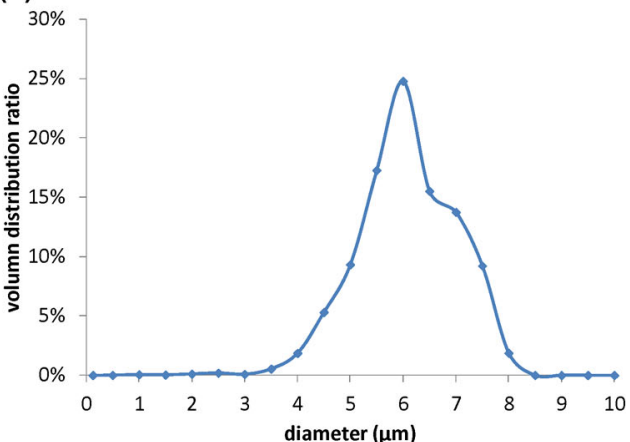

Figure 3. SEM images of PUMC synthesized in DMF in the presence of a) $0.3 \mathrm{v} / \mathrm{v} \%$ Span 85 , empty microcapsules; b) $0.1 \mathrm{v} / \mathrm{v} \%$ Span 85 , empty microcapsules; and c) $0.3 \mathrm{v} / \mathrm{v} \%$ Span 85 , cortisone loaded microcapsules. d) Diameter distribution of cortisone loaded microcapsule by volume based on 200 measurements. 
Span 85 onto the shells during the reaction, since the hydroxyl group on Span 85 could react with the residue isocyanate. Support to this hypothesis was lent by the finding that PUMC synthesized without Span 85 was hydrophilic and could be easily dispersed and swollen in water. Similar results had been previously reported using Span 80, another sorbitan-based surfactant. ${ }^{[19]}$ It was clear that non-ionic surfactants were capable of consuming the residue isocyanate on the shell and grafting themselves onto the surface of PUMC, resulting in a hydrophobic surface and poor water dispersibility. Furthermore, exposure to air (i.e., moisture) or a trace amount of nucleophilic agent is unavoidable in the post-modification and may significantly reduce the isocyanate group density on the surface. Therefore, post-modification of PUMC may not be an optimal strategy for introducing antibacterial functions because active isocyante groups could be lost prior to a secondary grafting reaction, leading to an insufficient surface modification. In the meantime, grafting of Span 85 onto PUMC demonstrates the potential of grafting a cationic antibacterial surfactant onto PUMC shell during its synthesis.

\subsection{One-Step Synthesis of Inherently Antibacterial Polyurea Microcapsules (MCQ)}

Inspired by the fact that surfactant Span 85 was covlantly bonded to the microcapsule shell during the synthesis, we attempted to synthesize an antibacterial surfactant DAB (Scheme 1) and to graft it onto the shell of PUMC in one step. The hydrophobic tail of DAB allowed its preferential adsorption at the interface of droplets to minimize interfacial tension. As an antimicrobial agent, DAB possesses an optimal bactericidal property since its carbon chain length reaches twelve. ${ }^{[20]}$ In addition, the hydroxyl group enables DAB to anchor onto the exterior surface of the microcapsules during the crosslinking reaction. Further, since DAB competes with PEI in reacting with TDI, we expected a reduced crosslinking density of the polyurea shell, which could lead to easier diffusion of TDI, higher PEI conversion rate, thicker shells and stronger microcapsules. Scheme 3 shows a schematic of the proposed in situ functionalization of microcapsule by encapsulating DAB into the disperse phase.

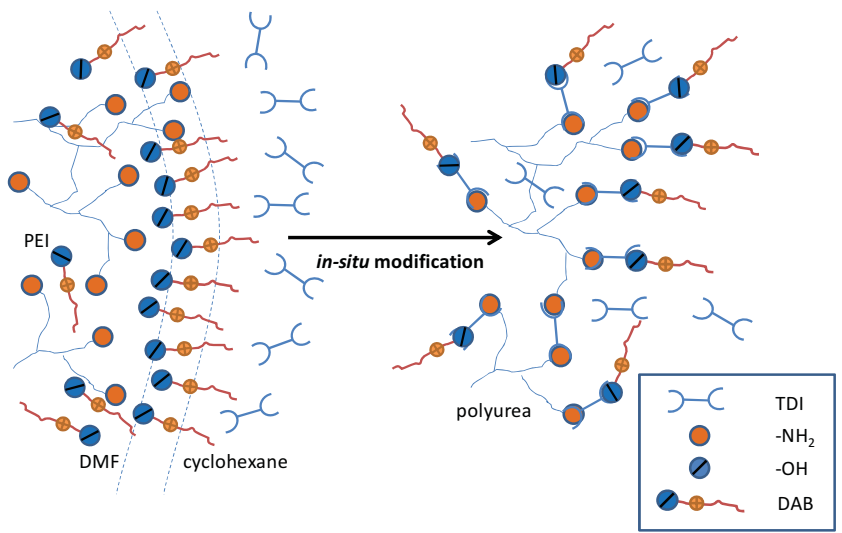

Scheme 3. In situ modification method (TDI: 2,4-tolylene diisocyanate; DAB: dimethyl-dodecyl-(5-hydroxy-pentyl)-ammonium bromide)).

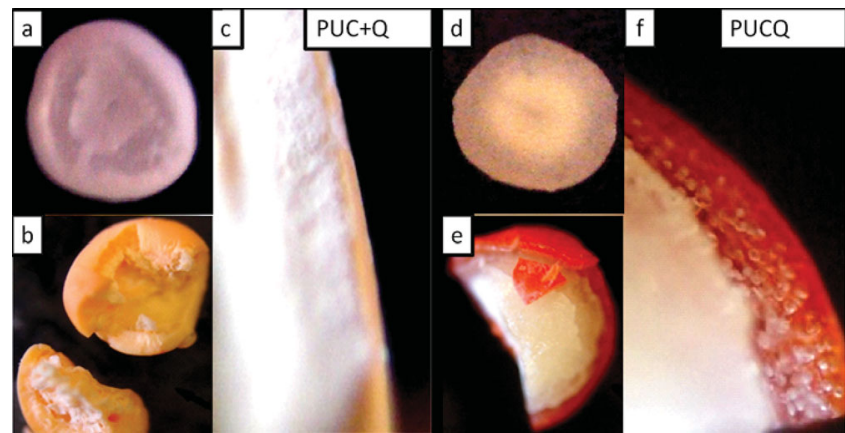

Figure 4. Cross-section of fluorescein stained model macrocapsules Left: post-modified model macrocapsules; right: in-situ modified model macrocapsules: a,d) freshly prepared model macrocapsules; b,e) cracked model macrocapsules after CTAC extraction; and c,f) close-up of fluorescein stained shell.

Again, we first synthesized in-situ modified model macrocapsule in which case DAB was added into the disperse phase before macrocapsule synthesis (addition of TDI into the continueous phase). The resultant PUC was referred to as "PUCQ". The DAB density on PUCQ was titrated with fluorescein and also presented in Figure 2. Fluorescein-stained PUCQ showed a much deeper color than stained PUC+Q, which indicated a higher amount of fluorescein in the shell (Figure 2 inset). However, no color change was observed during CTAC extraction and apparent DAB density of PUCQ $\left(5.9 \times 10^{-8} \mathrm{~mol} \mathrm{~cm}^{-2}\right.$, by subtracting PUCQ with PUC) was lower than that of PUC+Q $\left(4.3 \times 10^{-7} \mathrm{~mol} \mathrm{~cm}^{-2}\right.$, by subtracting PUC+Q with PUC). In order to elucidate these results, the cross-sections of capsules were studied under the microscope (Figure 4). Fluorescein molecules not only presented on the surface of the microcapsule, but also penetrated into the shell. The high DAB density and deeper penetration of fluorescein into the shell accounted for the unsuccessful desorption of negatively charged fluorescein from PUCQ. Because of this, the apparent DAB density data for PUCQ (Figure 2) is not the "true" representation of the density of DAB. After all, deeper fluorescein penetration into the shell of PUCQ than that of PUC+Q supports the hypothesis that DAB reduces crosslinking density of polyurea shell, leading to easier penetration of small molecules through the shell.

Apparent DAB density on PUCQ $\left(5.9 \times 10^{-8} \mathrm{~mol} \mathrm{~cm}^{-2}\right)$ was underestimated due to incomplete desorption of fluorescence in the test, but it still exceeded the threshold surface quaternary ammonium density for effective antibacterial surface $\left(0.8 \times 10^{-8} \mathrm{~mol} \mathrm{~cm}^{-2}\right) \cdot{ }^{[21]}$ The actual DAB density in the shell of PUCQ surpassed that of PUC+Q based on fluorescein color depth. Since PUCQ possessed better physiochemical properties than $\mathrm{PUC}+\mathrm{Q}$ in terms of shell thickness, rigidity, and DAB density, in-situ modification method was adopted to synthesize DAB modified PUMC. When DAB was added into DMF disperse phase to replace the surfactant Span 85 , the resultant PUMC was referred to as "MCQ". When PUMC was synthesized without either Span 85 or DAB, it was referred to as "MC" and served as a control sample for MCQ.

Table 2 lists the disperse phase composition of MCQ and MC. As shown in Figure 5, MCQ possessed thick shells (Figure 5a) and retained the spherical shape even after drying (Figure 5b). 
Table 2. Composition of disperse phase for synthesis of microcapsules.

\begin{tabular}{|c|c|c|c|c|c|}
\hline \multirow[t]{2}{*}{ Sample } & \multirow[t]{2}{*}{$\mathrm{Phi}^{\mathrm{a})}$} & \multicolumn{3}{|c|}{$\begin{array}{l}\text { Concentration of disp.phase } \\
{\left[\mathrm{mg} \mathrm{mL}^{-1}\right]}\end{array}$} & $\begin{array}{l}\text { Volume of TDI } \\
\qquad[\mathrm{mL}]\end{array}$ \\
\hline & & \multirow{2}{*}{$\begin{array}{c}\text { PEI } \\
50\end{array}$} & \multirow{2}{*}{$\frac{\text { DAB }}{100}$} & \multicolumn{2}{|c|}{ Coumarin-1 } \\
\hline MCQ-Cmr & $3.8 \%$ & & & 133 & 0.330 \\
\hline MCQ & $3.8 \%$ & 50 & 100 & 0 & 0.330 \\
\hline MC & $3.8 \%$ & 50 & 0 & 0 & 0.330 \\
\hline
\end{tabular}

a) $\mathrm{phi}=$ disperse phase volume/contineous phase volume $\times 100 \%$. The disperse phase solvent is anhydrous dimethylformamide, the contineous phase solvent is cyclohexane. with a typical release character of a reservoir-type drug delivery system, in which microcapsule shells regulate the permeation and diffusion of core reagents with a constant concentration gradient over an extended period of time. ${ }^{[22]}$ The shell thickness could be adjusted by changing DAB dosage, which further led to various specific release profiles of the core reagents.

To evaluate the inherent antimicrobial property, MCQ and the control microcapsule (MC) were extracted thoroughly to eliminate the potential interference from antibacterial chemical residues (e.g., TDI, PEI, ungrafted DAB, or methanol). The last batch of PBS extraction solution served as blank sample for antibacterial testing. Multi-drug-resistant extended-spectrum beta-lactamase Escherichia coli (MDR ESBL-E. coli) was chosen as a representative bacterium in the study.

In the agar diffusion test, a suspension

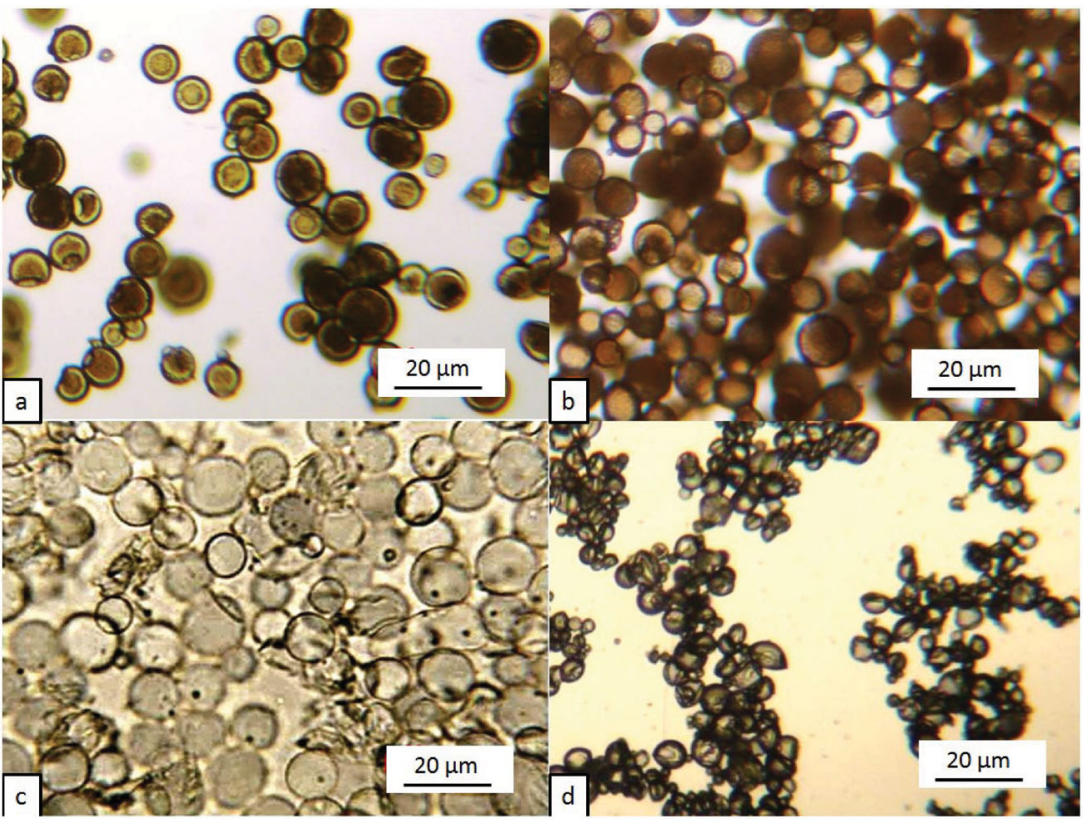

Figure 5. In situ modified polyurea microcapsule (MCQ) and the control polyurea microcapsule (MC). a) MCQ dispersed in water; b) dry MCQ; c) MC dispersed in water; and d) dry MC. of microcapsule was placed onto E. coli inoculated agar creating an area covered with microcapsules (microcapsule density: $0.88 \mathrm{mg} \mathrm{cm}{ }^{2}$, bacterial load: $4.54 \log _{10}$ CFU $\mathrm{cm}^{-2}$ ). As shown in Figure 7, a clear zone of inhibition was observed within MCQ covered area (diameter $=13.7 \mathrm{~mm}$ ). Within the $\mathrm{MC}$ covered area (diameter $=13.7 \mathrm{~mm}$ ), colonies spread around with only an altered shape, indicating that MCQ possessed an effective antibacterial property. There was no measureable expansion of inhibition zone outside the MCQ covered region and blank sample did not show any inhibitive effect on the colony. These findings confirmed that the observed antimicrobial effect of MCQ was due to the surface grafted DAB rather than DAB leached from MCQ.

Chronic wounds are a worldwide health problem and very costly to health-care. There is no one single dressing for all chronic wounds; however, dressing materials need
This property is critical in further handling and examining of the MCQ microcapsules. On the other hand, MC possessed thin soft shells and swelled significantly in water, indicating highly crosslinked primary membranes and hindered in-depth diffusion of TDI. Besides, MC had a larger diameter distribution $\left(\mu_{\mathrm{g}}=18.0 \mu \mathrm{m}, \mathrm{S}=0.69, \sigma_{\mathrm{g}}=1.27\right)$ than that of MCQ $\left(\mu_{\mathrm{g}}=\right.$ $\left.29.7 \mu \mathrm{m}, \mathrm{S}=0.53, \sigma_{\mathrm{g}}=1.21\right)$. Larger average diameter of MCQ probably resulted from the higher viscosity of MCQ dispersing phase caused by DAB, since highly viscous droplets were harder to be broken down to small droplets given the same emulsification condition.

MCQ was designed to function as both a drug carrier and an inherent biocide. To evaluate drug release property, coumarin-1, a hydrophobic model compound, was encapsulated into the microcapsule (referred to as "MCQ-Cmr") (recipe shown in Table 2). Figure 6 shows the drug release profile using USP Apparatus II. 35\% of drug loading was released within the first $24 \mathrm{~h}$ with a $8.5 \%$ initial burst release during the first $15 \mathrm{~min}$; the release curve stabilized at $2.14 \mathrm{mg} \mathrm{L}^{-1}$ on the $5^{\text {th }}$ day and $51.8 \%$ total drug loading was released within 7 days. The results fit

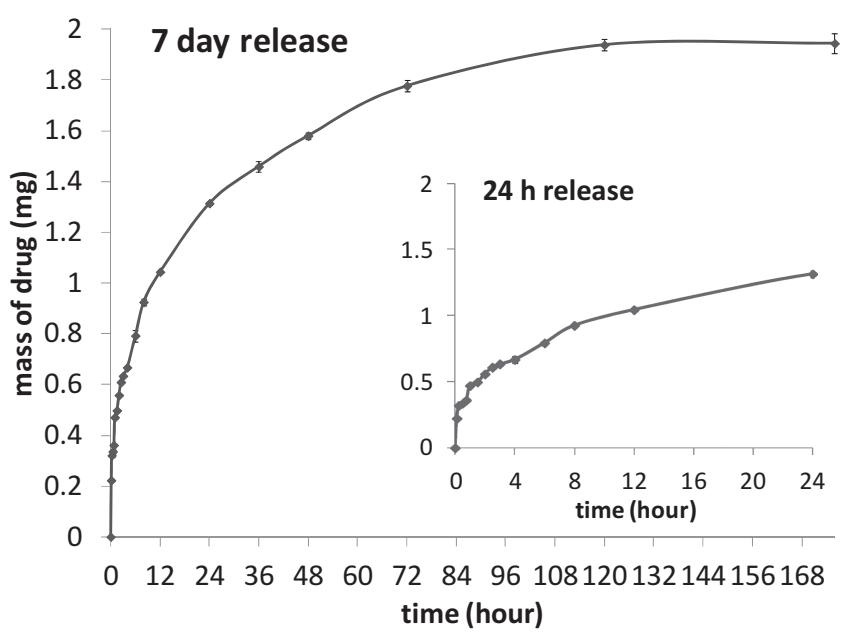

Figure 6. Seven-day in vitro cumulative release of coumarin-1 from MCQ$\mathrm{Cmr}$. Inset: first $24 \mathrm{~h}$ release curve. Drug load $=3.33 \mathrm{wt} \%$; efficiency $=$ $31 \%$. 


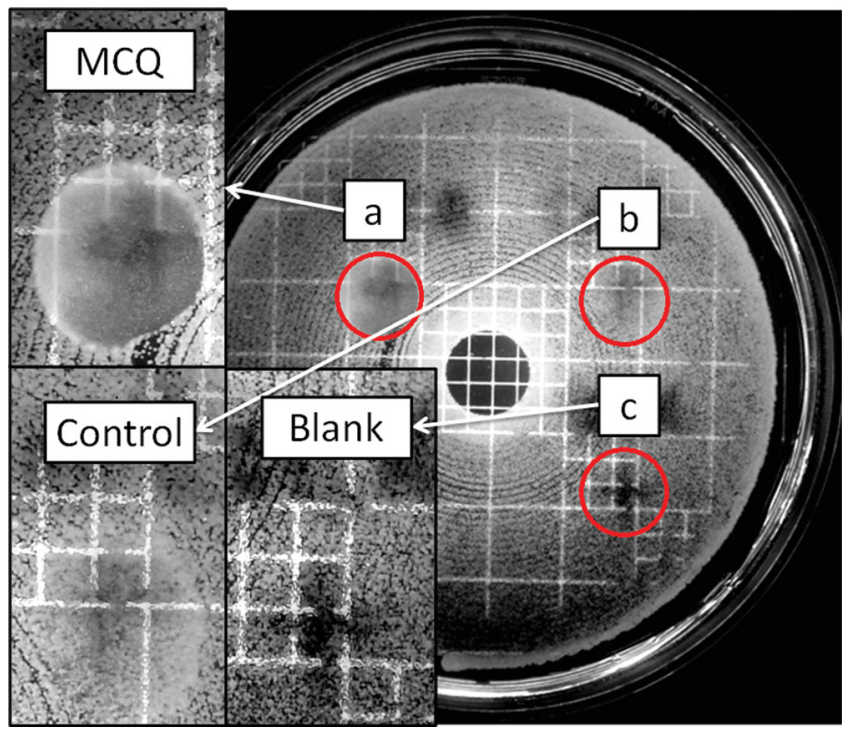

Figure 7. Agar diffusion test. A $10 \mu \mathrm{L}$-droplet of capsule suspension was dropped onto inoculated agar. Pictures were taken after $20 \mathrm{~h}$ incubation at $37^{\circ} \mathrm{C}$ : a) MCQ, DAB modified microcapsule; b) MC, control polyurea microcapsule without $D A B$ modification; and c) blank, last batch of washing solution of MCQ sample. Bacterial load: $4.54 \log _{10}$ CFU cm$~^{-2}$; microcapsule density: $0.88 \mathrm{mg} \mathrm{cm}^{-2}$.

both active wound infection control, and sustained topical delivery of nutrition or endogenous growth factors. MCQ could potentially be deposited onto the surface of chronic wound dressings to deliver both the functions of infection control and nutrition delivery. To simulate a gel-type dressing, MC and MCQ were evenly deposited onto tryptone soya agar (TSA) plates which were later inoculated with E. coli suspension. The results are shown in Figure 8 where white dots are colonies. Given a bacterial load of $3.54 \log _{10} \mathrm{CFU} \mathrm{cm} \mathrm{cm}^{-2}$, there was a total $1.97 \times 10^{5} \mathrm{CFU}$ on the agar plate $(8.5 \mathrm{~cm}$ in diameter). On the agar plates coated with MCQ, there was a clear trend of decreasing colonies with increasing densities of MCQ (0.22, 0.44, 0.66, $\left.0.88 \mathrm{mg} \mathrm{cm}^{-2}\right)$ : countless, countless but with less colonies, 96 and 24 colonies, respectively. To rule out the possibility that the decreased colonies may have resulted from less accessible agar nutrient to bacteria, the same amount of MC was coated onto agar plates: colonies were countless, independent of numbers of microcapsule layers (1-4 with corresponding densities of $0.22,0.44,0.66,0.88 \mathrm{mg}$ microcapsules $\mathrm{cm}^{-2}$, respectively). MC sample demonstrated no inhibition effect except that it caused the bacteria to spread over the microcapsules as the density of MC increased. The overspreading of bacterial colonies even on the agar plated coated with 0.66 or $0.88 \mathrm{mg} \mathrm{MC} \mathrm{cm}{ }^{-2}$ excluded the less intimate contact with nutrient agar at higher density of MCQ coating as a major reason for bacterial inactivation. The antibacterial action apparently resulted from the quaternary ammonium moiety of DAB that either penetrated the cell membrane or induced cation exchange compromising membrane integrity and inducing cellysis. ${ }^{[23,24]}$ Again, the bacterial killing was exerted through intimate contact with the antibacterial shell of MCQ instead of leaching biocides since the bacterial cells attached to MCQ surfaces were inactivated while those attached to the interspaces between microcapsules could still multiply, thus explaining why fewer colonies presented on the more densely coated MCQ agar plate.

These dual-functional inherently antibacterial microcapsules would have good potential for applications in medical and agricultural fields. Equally as import, the reported one-step surface modification strategy of adopting functional surfactants can be extended to other types of microcapsules synthesized by an interfacial polyaddition reaction such as polyurethane microcapsules, and provides polymer chemists with a useful tool to conveniently surface engineer polyurea/polyurethane microcapsules.

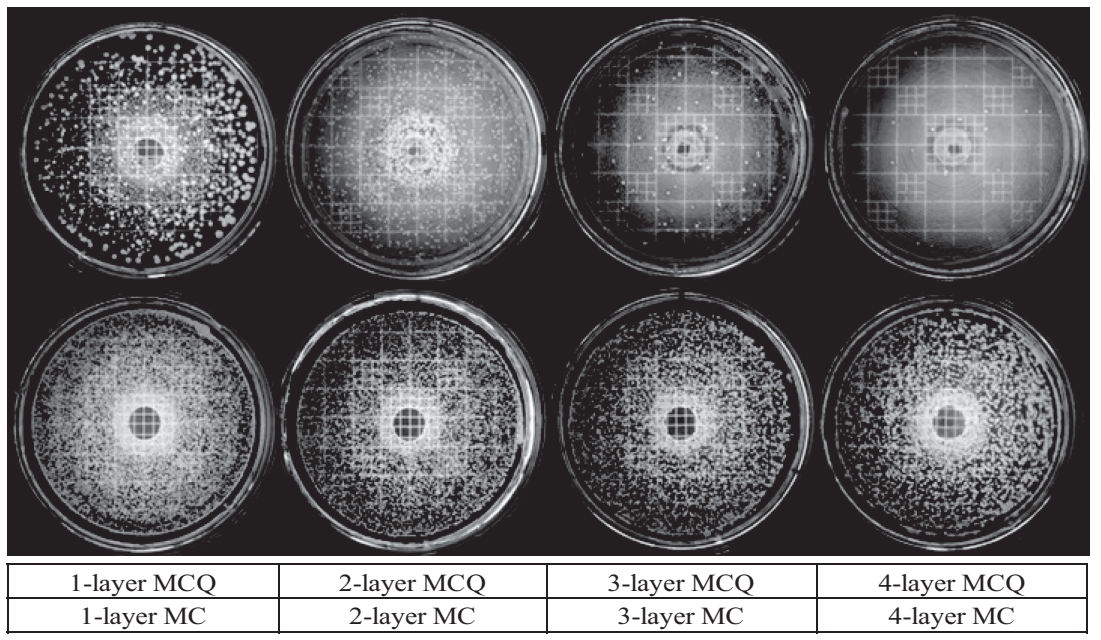

Figure 8. Static contact antibacterial test. Bacteria load: $3.54 \log _{10} \mathrm{CFU} \mathrm{cm} \mathrm{cm}^{-2}$; For each layer, $0.22 \mathrm{mg} \mathrm{cm}^{-2}(3.2 \mathrm{mg})$ capsules were coated over TSA in $100 \mathrm{~mm}$ petri dish using a spiral autoplater. MCQ: DAB modified polyurea microcapsule; MC: control polyurea microcapsule without DAB modification.

\section{Conclusions}

In situ antibacterial modification of polyurea microcapsules was successfully achieved at room temperature by using a new hydroxylended quaternary ammonium salt, DAB, in a surfactant-free oil-in-oil synthesis system. The synthesized microcapsules, MCQ, did not aggregate and were easily dispersible in water for further analysis and various applications. Furthermore, the prepared MCQ possessed both effective bactericidal and sustained drug release properties. This in-situ modification of microcapsules exemplified a unique way of incorporating a functional monomer into a PU shell while eliminating the need of extra surfactant, i.e. a facile modification of PU microcapsules in a cleaner system.

Antibacterial functionality is just one example of the many possible functions we 
can endow onto the shell of PUMC. We believe this study provides a universal strategy for the convenient and facile synthesis of new multifunctional PUMC, facilitating wider applications of PUMC in agricultural and biomedical fields.

\section{Experimental Section}

Reagents: Polyethylenimine branched (PEI, avg. $\mathrm{Mw}=25,000$ by LS, avg. $\mathrm{Mn}=10,000$ by GPC), 7-diethylamino-4-methylcoumarin (coumarin-1, a model drug), 2,4-tolylene diisocyanate (TDI) and Span 85 (Sorbitane trioleate, surfactant) were purchased from Sigma-Aldrich (Oakville, ON); the PEI solution in anhydrous dimethyformamide (DMF) was dried over molecular sieves (4 A) overnight before use. (Dimethylamino) pentan-1-ol was purchased from Karl Industry (Aurora, $\mathrm{OH}$ ), and 17-alpha,21-dihydroxypregn-4-ene-3,11,20-trione (cortisone, a model drug) was purchased from Tokyo Chemical Industry $(\mathrm{TCl})$ America (Portland, OR). All other reagents were purchased from SigmaAldrich (Oakville, ON) and were used as received. MDR ESBL-E. coli was obtained from the CANWARD (Canadian Ward Surveillance) study assessing antimicrobial resistance in Canadian hospitals (www.canr.ca).

Synthesis of AEA: AEA was synthesized according to a previous report. ${ }^{[25]}$ Briefly, sodium azide (3.6 g) was added to 2-bromoethylamine hydrobromide aqueous solution $\left(30 \mathrm{~mL}, 0.167 \mathrm{~g} \mathrm{~mL}^{-1}\right)$. The mixture was stirred overnight at $75{ }^{\circ} \mathrm{C}$. After the reaction mixture was cooled to room temperature, $\mathrm{NaOH}(976 \mathrm{mg}$ ) was added. The solution was further extracted with $\mathrm{CH}_{2} \mathrm{Cl}_{2}(3 \times 50 \mathrm{~mL})$. The combined organic extracts were then washed with saturated $\mathrm{NaCl}$ solution. The organic phase was dried over anhydrous $\mathrm{Na}_{2} \mathrm{SO}_{4}$. After filtration, the organic solvent was removed using a rotary evaporator to yield a clear, light-yellow liquid of 2-azidoethylamine $(1.85 \mathrm{~g}, 88 \%)$ that required no further purification. ${ }^{1} \mathrm{H}$ NMR $\left(400 \mathrm{MHz}, \mathrm{CD}_{3} \mathrm{SOCD}_{3}\right): \delta 3.21\left(\mathrm{t}, \mathrm{J}=6 \mathrm{~Hz}, 2 \mathrm{H} ; \mathrm{CH}_{2} \mathrm{~N}_{3}\right)$, $2.67\left(\mathrm{t}, \mathrm{J}=6 \mathrm{~Hz}, 2 \mathrm{H} ; \mathrm{CH}_{2} \mathrm{NH}_{2}\right), 1.44\left(\mathrm{~s}, \mathrm{br}, 2 \mathrm{H}, \mathrm{NH}_{2}\right)$. The structure of 2-azidoethylamine is shown in Scheme 1 .

Synthesis of DAB: To the solution of lauryl bromide (1.48 g, $5.94 \mathrm{mmol}$ ) in MeCN (15 mL) was added 5-(dimethylamino)pentan-7-ol $(0.72 \mathrm{~g}, 5.48 \mathrm{mmol})$, and the resulting solution was heated to reflux for $12 \mathrm{~h}$. Excessive solvent and lauryl bromide were removed under vacuum to yield the crude product, which was further purified on column chromatography eluting with $\mathrm{MeOH} / \mathrm{CH}_{2} \mathrm{Cl}_{2}(1: 1)$ to give $\mathrm{DAB}$ as a white solid. ' $\mathrm{H}$ NMR $\left(\mathrm{D}_{2} \mathrm{O}, 300 \mathrm{MHz}, \delta\right) 3.62\left(\mathrm{t}, J=6.7 \mathrm{~Hz}, 2 \mathrm{H}\right.$; $\left.-\mathrm{CH}_{2} \mathrm{OH}\right)$, 3.37-3.32 (m, $\left.4 \mathrm{H} ;-\mathrm{CH}_{2} \mathrm{~N}^{+} \mathrm{CH}_{2-}\right), 3.12\left(\mathrm{~s}, 6 \mathrm{H} ; \mathrm{N}^{+}\left(\mathrm{CH}_{3}\right)_{2}\right), 1.75-1.85(\mathrm{~m}$, $4 \mathrm{H}$; $-\mathrm{CH}_{2} \mathrm{CH}_{2} \mathrm{~N}^{+} \mathrm{CH}_{2} \mathrm{CH}_{2}-$, 1.68-1.59 (m, $2 \mathrm{H}$; $\left.-\mathrm{CH}_{2} \mathrm{CH}_{2} \mathrm{OH}\right)$, 1.31-1.49 ( $m, 20 \mathrm{H} ; \mathrm{CH}_{2}$ of lauryl chain), $0.91\left(\mathrm{t}, J=6.6 \mathrm{~Hz}, 3 \mathrm{H} ;-\mathrm{CH}_{2} \mathrm{CH}_{2} \mathrm{CH}_{3}\right.$ ); ${ }^{13} \mathrm{C}$ NMR $\left(\mathrm{D}_{2} \mathrm{O}, 75 \mathrm{MHz}, \delta\right) 64.5\left(-\mathrm{CH}_{2} \mathrm{CH}_{2} \mathrm{~N}^{+} \mathrm{CH}_{2} \mathrm{CH}_{2}-\right), 62.9\left(-\mathrm{CH}_{2} \mathrm{OH}\right)$, $32.3\left(-\mathrm{CH}_{2} \mathrm{CH}_{2} \mathrm{OH}\right), 29.6,29.5,27.3,24.2,22.7,20.4$, (29.6 to $22.4, \mathrm{CH}_{2}$ in the lauryl and pentyl alcohol chains), $14.2\left(-\mathrm{CH}_{2} \mathrm{CH}_{2} \mathrm{CH}_{3}\right)$; HRMS (MALDI-TOF) $\mathrm{m} / \mathrm{z}$ : [M-Br] ${ }^{+}$calculated for $\mathrm{C}_{19} \mathrm{H}_{42} \mathrm{NOBr}, 300.3266$; found, 300.3253. Structure of DAB is shown in Scheme 1.

Synthesis of PUC: PUC was synthesized to facilitate the study of surface chemistry of synthesis and to evaluate modification methods. Disperse phase $\left(10 \mu \mathrm{L}\right.$, typically a $50 \mathrm{mg} \mathrm{mL}^{-1} \mathrm{PEI}$ solution in DMF) was carefully injected into a microcentrifuge tube $(2.0 \mathrm{~mL}$ capacity, round bottom) containing $1 \mathrm{~mL}$ of anhydrous cyclohexane. DMF phase formed a single droplet at the bottom of the tube. Cyclohexane solution of TDI $(10 \mu \mathrm{L}, 25 \%, v / v)$ was carefully added to the cyclohexane phase without disturbing the DMF droplet at the bottom. Polymerization occurred instantly at the interface of the droplet, forming a microcapsule. The tube was gently vibrated and let stand for 5 min to allow for further diffusion of TDI. The resultant microcapsules were ready for further post-grafting reaction if necessary. Otherwise, the system was quenched with various amounts of ethanol/hexane solution after reaction.

Preparation of $P \cup C+Q$ : After the synthesis of PUC, the supernatant was carefully removed by pipetting, and DAB solution (typically $5 \mathrm{mg} \mathrm{mL} \mathrm{m}^{-1}$ ) in anhydrous tetrahydrofuran was added immediately. After the microcentrifuge tube was gently vortexed for $5 \mathrm{~min}$, the tetrahydrofuran solution of DAB was removed and microcapsules were washed with methanol repeatedly to remove loosely adsorbed DAB. Microcapsules were further extracted with methanol for another $48 \mathrm{hr}$ and dried to yield PUC+Q. PUC with residue isocyanate groups completely quenched with hexane solution of ethanol $\left(1.4 \times 10^{-4} \mathrm{~mol}\right.$ $\mathrm{L}^{-1}$ ) was also treated with tetrahydrofuran solution $\mathrm{DAB}$ as described above to serve as a control sample for PUC+Q.

Quantification of Residue Isocyanate Group on the Shell of PUC: Isocyanate groups on PUC were quantified using azide-labelling method. An IR-active compound with primary amine, AEA, was synthesized to serve as a labelling or reporting agent, which could be covalently grafted onto the microcapsules through a reaction with residue isocayante groups on the shell of PUC. After the freshly prepared PUCs were partially quenched with ethanol/hexane solution for $1 \mathrm{~min}$, they were added into AEA solution (typically $20 \mu \mathrm{L}, 10 \%, v / v$, in anhydrous tetrahydrofuran) for labelling. After 2 min of contact with gentle agitation, PUCs were washed with methanol repeatedly and were subjected to infrared spectra examination using $\mathrm{KBr}$ pellet method. In the case of the control sample, the system was quenched with pure ethanol for $20 \mathrm{~min}$ to ensure maximum quenching effect. After baseline correction, the characteristic band of azide group $\left(2108 \mathrm{~cm}^{-1}\right)$ was normalized with Amide I band $\left(1651 \mathrm{~cm}^{-1}\right) \cdot[26]$ Data obtained reflected the relative amount of AEA grafted on PUC.

Quantification of DAB density on the shell of PUC: DAB density, which is presented as the amount of fluorescein bound to PUC per unit surface area, was measured using fluorescein titration method. PUCs were immersed in fluorescein solution $(1 \mathrm{~mL} 1 \mathrm{wt} \%)$ for $5 \mathrm{~min}$ under gentle vibration. After removal of fluorescein solution, PUCs were thoroughly washed with Borex buffer $(0.1 \mathrm{M}, \mathrm{pH}=9.5)$. The microcapsules were then extracted with CTAC solution $(1 \mathrm{~mL} 0.1 \mathrm{wt} \%$, containing $0.1 \mathrm{M}$ Borex buffer, $\mathrm{pH}=9.5$ ) for three times under gentle agitation. The resultant solution of desorbed fluorescein was measured with UV-vis spectroscopy at $500 \mathrm{~nm}$.

Synthesis and Characterization of PUMC: PUMC was synthesized following the emulsion interfacial polymerization method ${ }^{[12]}$ with modifications. Briefly, disperse phase $(0.6 \mathrm{~mL}$, typically a $50 \mathrm{mg} \mathrm{mL}$ PEI solution in DMF) was injected into viscous cyclohexane $(15 \mathrm{~mL}$, poly(isobutylene) cyclohexane solution, $7.38 \mathrm{wt} \%, 158.9 \mathrm{CP}$, with typically $0.3 \mathrm{v} / \mathrm{v} \%$ Span 85 ) in a $30 \mathrm{~mL}$-beaker. The system was sealed and agitated with a magnetic flea at $1200 \mathrm{rpm}$ for $10 \mathrm{~min}$ to obtain an emulsion. TDI was added immediately after emulsification leading to instant formation of microcapsules. The system was agitated at $500 \mathrm{rpm}$ for another 2 min to allow for further diffusion of TDI into microcapsules. After the reaction, the system was diluted to $50 \mathrm{~mL}$ with ethanol/hexane solution $(10 \%, v / v)$ and was centrifuged at $1000 \mathrm{rpm}(\mathrm{rcf}=120 \mathrm{~g})$ for $10 \mathrm{~min}$. The microcapsules at the bottom were subjected to repeated washing with ethanol/hexane solution ( $25 \% \mathrm{v} / \mathrm{v}, 50 \% \mathrm{v} / \mathrm{v}, 75 \% \mathrm{v} / \mathrm{v})$ and pure ethanol to remove poly(isobutylnene) and TDI residues.

Size of the microcapsules was measured manually based on a microscopy image; particle size distribution (PSD) was calculated based on a method discribed by Merkus. ${ }^{[27]}$ Briefly, digital pictures were taken with a calibrated camera under an optical microscope. The diameters of over 200 microcapsules (PUMC $=401, M C=345, M C Q=226)$ were measured. Size distribution was represented by geometric standard deviation of diameters $\left(\sigma_{g}\right):\left(\sigma_{g}=D_{84} / D_{50}\right)$; and by Span value $(S): S=$ $\left(D_{90}-D_{10}\right) / D_{50}$, where $D_{n}$ represents diameter at $n \%$ point of cumulative undersize PSD based on volume weighted distribution. Average diameter was presented as volume weighted mean diameter $\mu_{\mathrm{g}}=\mathrm{D}(4,3)=\sum \mathrm{n}_{\mathrm{i}}$ $\mathrm{D}_{\mathrm{i}}^{4} / \sum \mathrm{n}_{\mathrm{i}} \mathrm{D}_{\mathrm{i}}{ }^{3}$.

Dissolution Test: In vitro drug release of coumarin-1 from MCQ was tested using USP Apparatus II on a VanKel 600 Dissolution Apparatus (Palo Alto, CA, USA). ${ }^{[28]}$ Briefly, approximately $20 \mathrm{mg}$ MCQ containing coumarin- 1 was accurately weighed and dispersed in $250 \mathrm{~mL}$ PBS $(0.1 \mathrm{M}$, $\mathrm{pH}=7.4)$. The paddle speed was set at $75 \mathrm{rpm}$ and the test temperature at $37 \pm 0.5^{\circ} \mathrm{C}$. At predetermined time intervals $(0.25,0.5,0.75,1,1.5,2$, $2.5,3,4,6,12,18,24,36,48,72,120$, and $176 \mathrm{~h}$ ), $1.0 \mathrm{~mL}$ suspension was filtered through a $0.2 \mu \mathrm{m}$ filter and was subjected to HPLC-UV measurement. $1.0 \mathrm{~mL}$ fresh PBS solution was then replenished to the dissolution vessels. The HPLC-UV analysis was conducted using 
a Waters Separation Module equipped with a PDA detector and a $\mathrm{C}_{18}$ column ( $\mu$ Boundapak C-18 $10 \mu \mathrm{m}$ 125A). Chromatographic conditions were as following: mobile phase: $70 \% \mathrm{MeCN}$ and $30 \% \mathrm{H}_{2} \mathrm{O}$; flow rate: $1.0 \mathrm{~mL} \mathrm{~min}{ }^{-1}$; retention time: $2.45 \mathrm{~min}$; and absorbance: $380 \mathrm{~nm}$, and detection limit $\left(0.1 \mathrm{mg} \mathrm{L}^{-1}\right)$.

Antibacterial Tests: The stock suspension of MC or MCQ $\left(40 \mathrm{mg} \mathrm{mL}^{-1}\right.$, in sterile $0.1 \mathrm{~mol} \mathrm{~L}^{-1}$ PBS solution, $\mathrm{pH}=7.4$,) was prepared by sequential extraction of microcapsules with methanol $(3 \times 10 \mathrm{~mL})$ and PBS $(3 \times 10 \mathrm{~mL}$, $48 \mathrm{~h}$ ) to thoroughly remove residue of DAB or methanol. The last batch of PBS extraction was collected to serve as blank sample for surface diffusion test. MDR ESBL-E. coli was cultured in MacConkey broth to mid-log phase and was diluted to desired concentrations with sterile PBS solution on site.

For the agar diffusion test, TSA agar plates were inoculated with E. coli suspension with Autoplate 4000 . Microcapsule suspension $(20 \mu \mathrm{L}$, $8.0 \log _{10} \mathrm{CFU} \mathrm{mL} \mathrm{mL}^{-1}$ ) was placed onto the surface of agar. The agar plates were subjected to incubation at $37^{\circ} \mathrm{C}$ for $20 \mathrm{~h}$.

For the static contact test, the microcapsule suspension was evenly coated onto the TSA agar surface with a spiral plater (Autoplate 4000). Multiple layers of microcapsule suspension were coated onto the agar surface at approximately 2 min intervals to allow for drying of the previous layer (for each layer, density of microcapsule: $0.22 \mathrm{mg} \mathrm{cm}^{-2}$ ). Agar plates were then inoculated with $E$. coli suspension $\left(20 \mu \mathrm{L}, 7.0 \log _{10}\right.$ $\mathrm{CFU} \mathrm{mL} \mathrm{m}^{-1}$ ) and were subjected to incubation at $37^{\circ} \mathrm{C}$ for $20 \mathrm{~h}$.

\section{Acknowledgements}

The authors acknowledge financial support from the Manitoba Health Research Council (MHRC, Operating and Establishment Grants), the Dr. Paul H.T. Thorlakson Foundation and the University of Manitoba Research Grant Program. The authors also gratefully acknowledge the technical assistance of Drs. Lingdong Li (organic synthesis), Rick Holley, and Saqer Herzallah (antibacterial testing).

Received: March 8, 2012

Published online:

[1] F. M. Veronese, F. Marsilio, S. Lora, P. Caliceti, P. Passi, P. Orsolini, Biomaterials 1999, 20, 91.

[2] Y. Wang, Z. Lu, H. Wu, F. Lv, Int. J. Food Microbiol. 2009, 136, 71.

[3] Q. Wang, J. Gong, X. Huang, H. Yu, F. Xue, J. Appl. Microbiol. 2009, 107,1781
[4] P. D. Bragg, D. J. Rannie, Can. J. Microbiol. 1974, 20, 883.

[5] R. B. Thurmann, C. P. Gerba, Crit. Rev. Environ. Control 1989, 18, 295.

[6] S. Silver, FEMS Microbiol. Rev. 2003, 27, 341.

[7] S. M. Modak, C. L. Fox, Arch. Surg. 1981, 116, 854.

[8] A. T. Hendry, I. O. Stewart, Can. J. Microbiol. 1979, 25, 915.

[9] D. Cui, A. Szarpak, I. Pignot-Paintrand, A. Varrot, T. Boudou, C. Detrembleur, C. Jérôme, C. Picart, R. Auzély-Velty, Adv. Funct. Mater. 2010, 20, 3303.

[10] I. El-Gibaly, A. Anwar, Int. J. Pharm. 2004, 278, 25.

[11] P. Ni, M. Zhang, N. Yan, J. Membr. Sci. 1995, 103, 51.

[12] M. Kobaslija, D. T. McQuade, Macromolecules 2006, 39, 6371.

[13] J. Hickey, N. A. D. Burke, H. D. H. Stöver, J. Membr. Sci. 2011, 369, 68.

[14] J. Li, M. A. J. Mazumder, H. D. H. Stöver, A. P. Hitchcock, I. M. Shirley, J. Polym. Sci. Part A: Polym. Chem. 2011, 49, 3038.

[15] B. P. Mason, M. H. Steven, G. F. Strouse, D. T. McQuade, Org. Lett. 2009, 11, 1479.

[16] J. Li, A. P. Hitchcock, H. D. H. Stöver, I. Shirley, Macromolecules 2009, 42, 2428.

[17] V. Schmitt, C. Cattelet, F. Leal-Calderon, Langmuir 2004, 20, 46.

[18] F. Thivilliers, N. Drelon, V. Schmitt, F. Leal-Calderon, Europhys. Lett. 2006, 76, 332

[19] G. Li, Y. Q. Feng, X. G. Li, P. Gao, J. Wang, J. Y. Xie, J. Mater. Sci. 2007, 42, 4838

[20] H. Murata, R. R. Koepsel, K. Matyjaszewski, A. J. Russel, Biomaterials 2007, 28, 4870

[21] L. Caillier, E. T. de Givenchy, R. Levy, Y. Vandenberghe, S. Geribaldi, F. Guittard, Eur. J. Med. Chem. 2009, 44, 3201.

[22] E. M. Valle, M. A. Galan, R. G. Carbonell, Ind. Eng. Chem. Res. 2009, $48,2475$.

[23] K. Page, M. Wilson, I. P. Parkin, J. Mater. Chem. 2009, 19, 3819.

[24] J. Lin, S. Qiu, K. Lewis, A. M. Klibanov, Biotechnol. Prog. 2002, 18, 1082.

[25] S. Angelos, Y. W. Yang, K. Patel, J. F. Stoddart, J. I. Zink, Angew. Chem. Int. Ed. 2008, 47, 2222

[26] K. Sahre, M. H. A. Elrehim, K. Eichhorn, B. Voit, Macromol. Mater. Eng. 2006, 291, 470.

[27] H. G. Merkus, Particle size measurements: fundamentals, practice, quality, Springer, Dordrecht 2009.

[28] T. Hickey, D. Kreutzer, D. J. Burgess, F. Moussy, Biomaterials 2002, 23, 1649. 